\title{
A BEHAVIOR BASED NONLINEAR MULTI-AGENT FRAMEWORK USING A HYBRID APPROACH
}

\author{
Chih-Fu Chang and Li-Chen Fu*
}

\begin{abstract}
In this paper, a novel design and implementation of a multi-agent system via a hybrid approach is proposed. First of all, an autonomous agent is insured to be stable by employing the proposed local feedback control besides appropriate switching among well-posed behaviors including obstacle avoidance, target approaching, wandering, manipulator grasping, etc. Next, a hybrid framework of the multi-agent system (HMAS) is established for dealing with multi-agent cooperative problems. The architecture of the inherent hierarchical control is designed consisting of two levels, where the first (lower) level mainly handles the continuous state describing the data acquired from various sensors, whereas the second (higher) level is responsible for providing a well performed supervisory control that can manipulate all available resources to successfully accomplish the underlying mission. In the Advanced Control Laboratory of the Department of Electrical Engineering at National Taiwan University, an actual wheeled mobile robot (WMR) has been developed, named The Treasure Hunter (TTH), which is able to hunt treasures in an unknown environment whereby the effectiveness of the proposed approach can be satisfactorily demonstrated.
\end{abstract}

Key Words: hybrid system, multi-agent system, wheeled mobile manipulator, visual tracking.

\section{INTRODUCTION}

The hybrid system using automation theory has been studied extensively in recent years. There have been several topics in this field which remain attractive to lots of scholars, such as liveness, reachability, stability, and controllability. Among those, stability of the discrete event system (DES) has been brought to attention since one of its important sub-topics which concerns the Lyapunov method (Ozvern and Willsky, 1991) and the output stability method (Passino et al., 1994) have become recent focuses of research interests. Also, Lyeros et al. (2003) have developed a more general theory on dynamic properties of a hybrid system. Linear Matrix Inequality (LMI) analysis has been used to prove the stability

*Corresponding author. (Email: lichen@ntu.edu.tw)

C. F. Chang is with the Department of Electrical Engineering, National Taiwan University, Taipei, Taiwan 106, R.O.C.

L. C. Fu is with the Department of Computer Science and Information Engineering, National Taiwan University, Taipei, Taiwan 106, R.O.C. of the hybrid systems (Zhang and Tarn, 2003).

In this paper, every single unit of the multi-agent system is considered to be a nonholonomic wheeled mobile robot (WMR). As a result, the typical problems of nonholonomic systems have to be taken into consideration when hybrid systems need to be properly designed particularly in the continuous-time domain. However, there are already several delicate research results in kinematic control of nonholonomic systems, such as a unified and accessible presentation of system models, problem formulations, solution approaches, and control results (Kolmanovsky and McClamroch, 1995), a suboptimal trajectory control using sinusoids at integrally related frequencies to achieve motion at a given bracketing level (Murray and Sastry, 1993), and a cyclic process for designing a nonholonomic controller (Brockett, 2003). Nevertheless, it seems to be more complex to deal with such a WMR equipped with a manipulator due to its excessive redundancy problem and somewhat intricate kinematic constraints from the viewpoint of the entire system's structure, as revealed by Altafini 
(2004), Korayem and Ghariblu (2003) and Kemeny (2003).

A multi-agent cooperative system (MAS) asks every agent to work with others cooperatively so as to solve a problem by using local expertise and resources first, and then by integrating these suboptimal solutions into an overall solution. Apparently, this problem is hybrid with different concerns from either the continuous-time domain or from the discrete event domain, such as modeling, interpretation, planning, and control. Durfee et al. (1989) have presented a detailed overview of a cooperative problem from the viewpoint of a discrete event system. On the other hand, Burgard and Moors (2005) have developed a selection and path planning method for a heterogeneous robot team to explore an unknown environment. Another approach is proposed by Kumar and Grag (2004) which is to deal with a cooperating movement problem with force control based WMR without considering kinematic constrains. Last but not least, communication is also a very important factor that leads to successful cooperation among WMRs (Nett and Schemmer, 2003).

The aim of this paper is to develop a hybrid system approach to realize a control architecture for a wheeled mobile manipulator (WMM) to execute a task of searching for a target and to grasp it while wandering through the environment cluttered with obstacles and to extend this to a multi-agent framework. This paper is organized as follows : The problem formulation and the pertaining system architecture of the WMM are presented in Section II and III, respectively. On the other hand, hybrid system modeling and control is introduced in Section IV. Different kinds of sub-systems are introduced in section $\mathrm{V}$. The setup of the overall system for experiment and extensive simulation results are provided in Section VI, and finally conclusions are drawn in Section VII.

\section{PROBLEM FORMULATION}

The hybrid multi-agent system (HMAS) is a kind of architecture which can address the distributed control problem for a number of agents, in which each is characterized by a hierarchical structure with hybrid continuous and discrete event states. As shown in Fig. 1, the illustrated hybrid system with supervisory control is designed to deal with various problems that may be encountered within the multi-agent framework. As a matter of fact, such system structure can also be regarded as a hierarchical architecture whose design challenge is to take care of switching control which couples continuous state constraints as well as supervisory control seeking to meet discrete state optimality of a multiagent system.

In this paper, there are several steps proposed

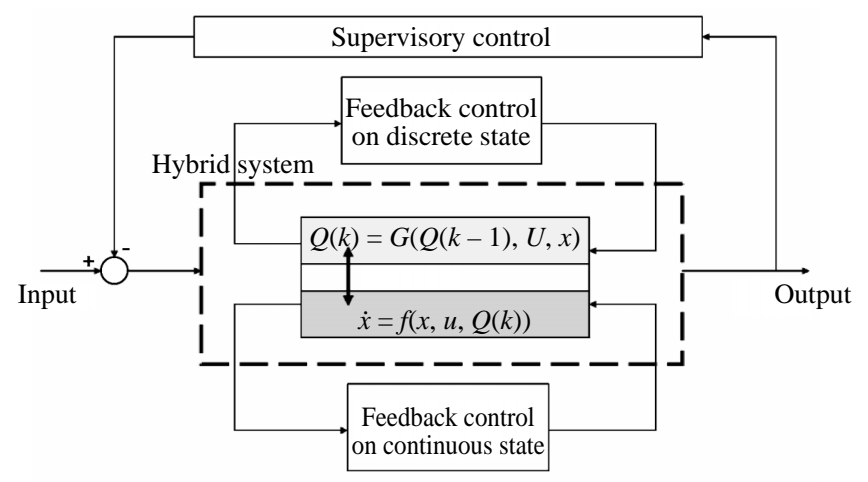

Fig. 1 Hybrid system with supervisory control

to design the underlying complex multi-agent system. First of all, we devise a single agent with a hybrid system nature which can switch between various local behaviors autonomously under different circumstances. However, such integrated control of a nonholonomic WMM resulting from switched behaviors is difficult to optimize and to analyze with traditional methods. To resolve this, we first adopt the nonholonomic control method proposed by Chang et al. 2004. Next, the major problem left in this paper is the framework design and implementation of the HMAS given that a stable control method is applied to each of the WMMs. The stability and controllability analysis of the proposed HMAS will be conducted in our future work.

Furthermore, at every discrete event state, the discrete constraint situation will not be particularly emphasized throughout the paper. In addition to this, to simplify the problem of communication among different agents, we here assume that a wireless network has been established over the entire area where the agents may move. Finally, this research work will choose a behavior based supervisory control to resolve the multi-agent problem in HMAS.

\section{SYSTEM ARCHETECTURE}

A WMM was built in Advanced Control Laboratory of the Department of Electrical Engineering at National Taiwan University, designed for target searching and grasping. Here, the mobile base is assumed to be equipped with wheel encoders, ultrasound sensors, a CCD camera, and a laser ranger finder. There are four features of this mobile manipulator, including: 1). novel mechanical design; 2). ability to explore unknown environment; 3 ). visual tracking system, 4). remote supervisor system.

As for the manipulator, which is mounted on the mobile base, it is a 5 degree-of-freedom (DOF) robot arm. Besides, the aforementioned ultrasonic sensing system employ six ultrasonic sensors aligned to six 


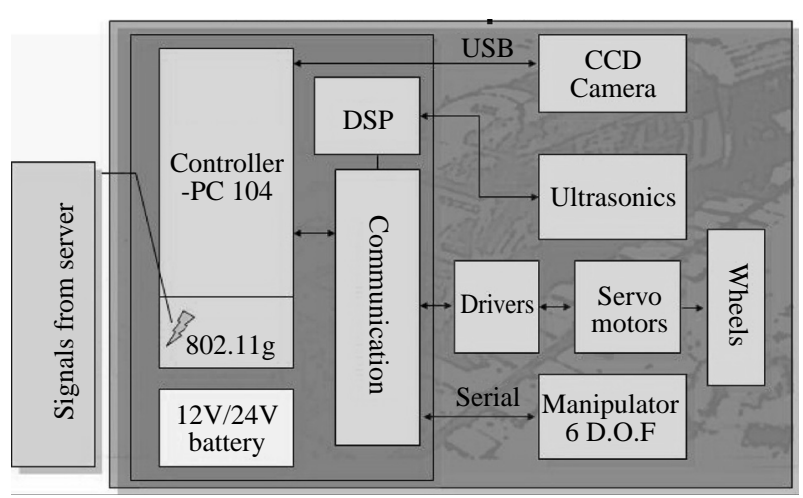

Fig. 2 System conceptual diagram

different directions and a DSP for signal processing in order to perform the task of collision avoidance. Fig. 2 displays the conceptual diagram of the entire system. There are two main processors installed in the WMM which are used to process the main jobs, including image processing, controller computation, and I/O access.

In the Advanced Control Lab of Dept. of Electrical Engineering at National Taiwan University, an actual WMM is developed, named The Treasure Hunter (TTH), which is designed to be one of an agent in an unknown environment. Fig. 3 displays the TTH system as the simulation and experimental test bed in this paper.

\section{HYBRID SYSTEM MODELING AND CONTROL}

In this section, we start with the pertinent hybrid system model. Before that, kinematic and dynamic models of the WMM, and its nonlinear controller design will be derived in the following.

\section{Dynamics Model of a WMM}

The Lagrange formulation is used to establish equations of motion for the WMM (Kemeny, 2003), which may lead to the following matrix form

$$
M(x) \ddot{x}+V(x, \dot{x}) \dot{x}+G(x)=E(x) \tau-A^{T}(x) \lambda
$$

where $x=q_{v} \otimes q_{m}$ is the overall system state consist-

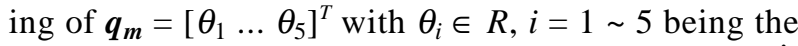
join variables of the manipulator and $\dot{\boldsymbol{q}}_{v}=\left[\begin{array}{lll}\dot{x}_{v} & \dot{y}_{v} & \dot{\phi}_{v}\end{array}\right.$ $\left.\dot{\varepsilon}_{v}\right]^{T}$ being the control variables of the mobile base $\left(\left(\dot{x}_{v}, \dot{y}_{v}\right)\right.$ is the linear velocity, $\dot{\phi}_{v}$ is the rotating speed, and $\dot{\varepsilon}_{v}$ is the forward moving speed), $M(x)$ is the inertia matrix, $V(x, \dot{x})$ is the state of centripetal and Coriolis torques, $G(x)$ is the gravity term, $A(x)$ is the relevant constraint matrix, and $\tau$ is a generalized control torque.

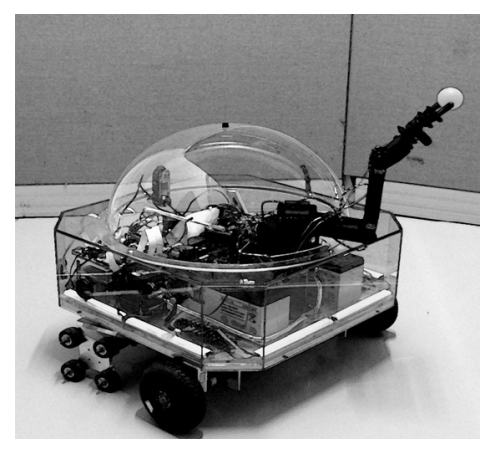

Fig. 3 The WMM, named The Treasure Hunter (TTH), developed at NTU

Proposition 1. (Stable Nonlinear Control, Chang et al. 2004): Consider the system described by Eq. (1) with the following control law :

$$
\begin{aligned}
\tau= & E^{+}(x)\left(M(x) \ddot{x}_{d}+V\left(x, \dot{x}_{d}\right) \dot{x}_{d}+G(x)+A^{T}(x) \lambda\right. \\
& \left.-K_{p} e-K_{d} \dot{e}\right)
\end{aligned}
$$

with the definitions :

$$
e=x-x_{d}, \dot{x}_{r}=\dot{x}_{d}-\Lambda e, s=\dot{x}-\dot{x}_{r}=\dot{e}+\Lambda e
$$

where $e$ is the state tracking error, $x_{d}, x_{r}, s \in R^{n}$ denote the desired state; reference state; and mixed error, respectively; $E^{+}(x)=\left(E(x)^{T} E(x)\right)^{-1} E(x)^{T}$ denotes a pseudo inverse matrix, $\lambda$ is a set of appropriately chosen parameters, and both $K_{p}$ and $K_{d}$ are positive definite constant matrices. Then, the WMM system is shown to be exponentially stable.

Corollary 1: From Proposition 1, the desired acceleration, $\ddot{x}_{d}$, can be transformed to the following control space

$$
\ddot{x}_{d}=K_{2}\left(\dot{J}\left(x_{d}\right) v_{d}+J\left(x_{d}\right) \dot{v}_{d}\right)
$$

for appropriately defined Jacobian matrix $J(\cdot)$ and $v_{d}$ $=\dot{x}_{d}$. Then, the generalized control torque defined as

$$
\begin{aligned}
\tau= & E^{+}(x)\left(\bar{M}(x) \dot{v}_{d}+\bar{V}\left(x, v_{d}\right) v_{d}+G(x)+A^{T} \lambda\right. \\
& \left.-K_{p} e-K_{d} \dot{e}\right)
\end{aligned}
$$

will ensure that the WMM system is exponentially stable, where $\bar{M}(x), \bar{V}\left(x, v_{d}\right)$ are appropriately defined versions of $M(x), V\left(x, v_{d}\right)$.

The proof is straightforward and the proposed control block is shown by Fig. 4. Actually, the implementation suffers from the difficulty of solving the inverse torque since we neglect the nonlinear actuator dynamics. In that case, the linear relationship between output 
Table 1 Finite set of discrete states

\begin{tabular}{cccccc}
\hline & \multicolumn{2}{c}{ Discrete state } & \multicolumn{2}{c}{$q_{i}$} & Discrete state \\
\hline$q_{0}$ & $W$ & Wandering & $q_{11}$ & $T F$ & Finely-tune \\
$q_{1}$ & $R_{1}$ & Reverse_1 & $q_{12}$ & $R_{2}$ & Reverse_2 \\
$q_{2}$ & $A_{v}$ & Avoid & $q_{13}$ & $W_{2}$ & Wait_2 \\
$q_{3}$ & $S$ & Searching & $q_{14}$ & $G$ & Grasp \\
$q_{4}$ & $G L$ & GoToLocation & $q_{15}$ & $W_{3}$ & Wait_3 \\
$q_{5}$ & $G A$ & GoToArea & $q_{16}$ & $T$ & Transportation \\
$q_{6}$ & $F M$ & Formation_Master & $q_{17}$ & $P$ & Park \\
$q_{7}$ & $F S$ & Formation_Slave & $q_{18}$ & $R_{3}$ & Reverse_3 \\
$q_{8}$ & $W_{1}$ & Wait_1 & $q_{19}$ & $S t$ & Start \\
$q_{9}$ & $A p p$ & Approach & $q_{20}$ & $E$ & End \\
$q_{10}$ & $T R$ & Roughly-tune & & & \\
\hline
\end{tabular}

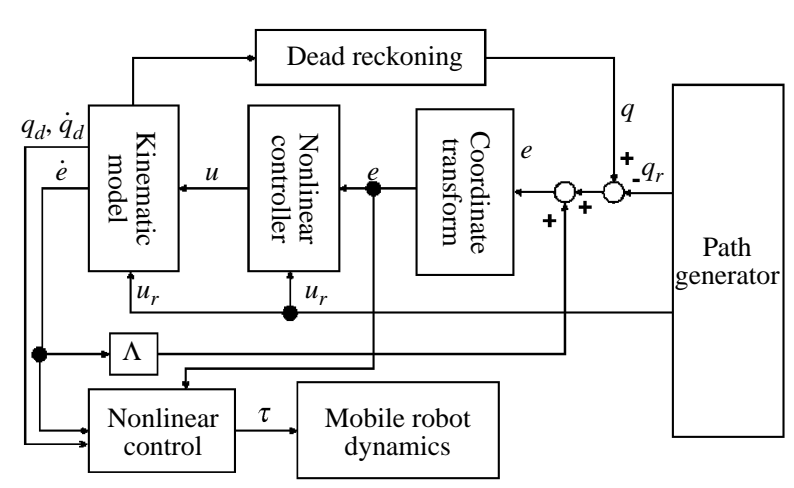

Fig. 4 Local nonlinear virtual torque control

velocity, torque and voltage are hereby assumed for simplicity of demonstrating the concept of this paper.

\section{Hybrid Automaton}

A hybrid automaton is a dynamic system that describes the evolution in time of the valuations of a set of discrete and continuous variables. In addition, there are several brilliant researches with dynamic property have been addressed such as Ozvern and Willsky (1991) and Lyeros et al. (2003). In this paper, a hybrid automaton designed for WMM is implemented to evaluate the reachability and stability issue. The defininitions, therefore, of variables on discrete states are depicted as Table 1 for helping reader's plain. More detail information on using these variables will be revealed in next section.

Definition 1. The hybrid automata, $H=\left(H_{1} \cdots H_{m}\right)$, of the multi-agent robot system is a collection of individual hybrid automaton $H_{i}=\left(Q_{i}, X_{i}, U_{i}, Y_{i}\right.$, Init ${ }_{i}$, $\left.f_{i}, \operatorname{Inv}_{i}, E_{i}, G_{i}, R_{i}\right), i=1, \cdots, m$, where:

$Q_{i}$ is a finite set of discrete states with the following meanings:

$$
\begin{aligned}
Q_{i}= & \left\{q_{0} \cdots q_{20}\right\} \\
= & \left\{S_{t}, W, S, G L, G A, F M, F S, W_{1}, A p p, A_{v},\right. \\
& \left.T R, T F, R_{1}, R_{2}, R_{3}, W_{2}, W_{3}, G, T, P, E\right\}
\end{aligned}
$$

$U_{i}=\left(v_{i}, s_{i}, W\right)$ is the set of input variables;

$Y_{i}=\left(c_{i}, o_{i}\right)$ is the set of output variables with $c$ and $s$ denoting the image measurement and the surrounding obstacle information (relative to the robot), respectively;

Init $_{i}$ is the set of initial conditions of the system, $\dot{X}_{i}=f_{i}\left(X_{i}\right)+f_{i}\left(q_{i}, v_{i}, s_{i}, W\right)$ is the state governing equation where $X_{i}$ is the continuous state vector defined as $X_{i}=\left[x_{i}, y_{i}, \phi_{i}, q_{m i}\right]^{T}$ $\in R^{8}, f(\cdot) \in R^{8}$ is the kinematics model of the WMM without considering extra input, $f_{i}: Q_{i} \times R^{9+(m-1)} \rightarrow R^{8}$ is a force field defined as :

$$
\begin{aligned}
f_{i} & =f_{i}\left(q_{i}, v_{i}, s_{i}, W\right) \\
& =f_{i}\left(q_{i}, I_{A}, I_{u}, I_{v}, S_{0}, S_{1}, S_{2}, S_{3}, S_{4}, S_{5}, W\right)
\end{aligned}
$$

where $v_{i}=\left\{I_{A}, I_{u}, I_{v}\right\} \in R^{3}$ denotes discrete variables after the CCD camera image is processed, $s_{i} \in R^{6} \mathrm{de}-$ picts the six distance measurements acquired from the set of six ultra-sonic sensors, and $W$ is the communication information from other $(m-1)$ robots,

$\operatorname{Inv} v_{i}$ is the domain (invariant set),

$E_{i}$ is the set of transitions.

$G_{i}$ is the guard-set function,

$R_{i}$ is the reset function.

Consequently, a hybrid automata is hereby adopted to model the multi-agent framework of multiple WMMs, and one of robots could just be the TTH. The conceptual flow diagram of the discrete states is depicted in Fig. 5.

Definition 2: The execution of a hybrid automation $H_{i}, i=1, \cdots, m$ is a collection of $\chi_{i}=\left(\gamma_{i}, q_{i}, x_{i}\right)$, where $\gamma_{i}$ is a hybrid time trajectory, $\left.q_{i}<\gamma_{i}\right\rangle \rightarrow Q_{i}$ is a discrete variable, and $x_{i}=\left\{x_{i}^{j} ; j \in\left\langle\gamma_{i}\right\rangle\right\}$ is a collection 


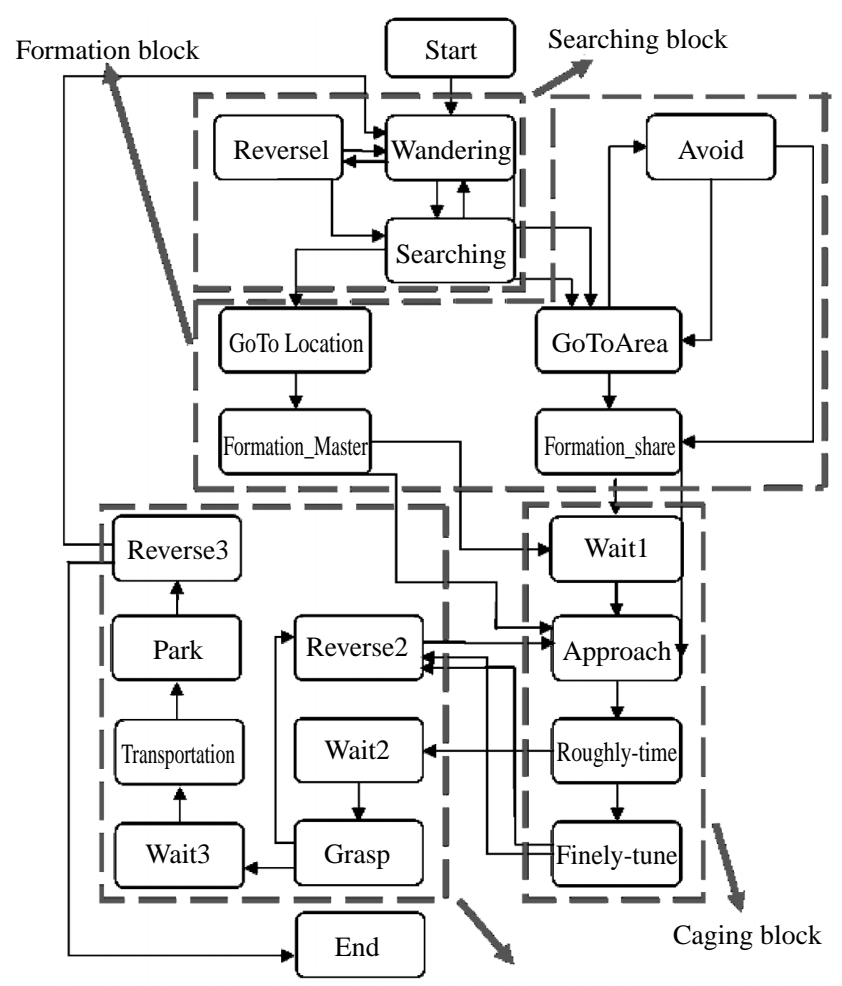

Fig. 5 Simple conceptual flow diagram of the discrete states

of dynamics such that

$$
\begin{aligned}
& \left(q_{i}(0), x_{i}^{0}(0)\right) \in \text { Init }_{i} ; \\
& \dot{x}_{i}^{j}=f_{i}\left(q_{i}(j), x_{i}^{j}(t)\right) \text { and } x_{i}^{j}(t) \in D\left(q_{i}(j)\right), \\
& \forall t \in\left[\tau_{j}, \tau_{j}^{\prime}\right) ; \\
& \forall j \in\left\langle\gamma_{i}>\{N\}, e_{i}=\left(q_{i}(j), q_{i}(j+1)\right) \in E,\right. \\
& x_{i}^{j}\left(\tau_{j}^{\prime}\right) \in G\left(e_{j}\right), \text { and } x_{i}^{j+1}\left(\tau_{j+1}\right) \in R\left(e_{i}, x_{i}^{j}\left(\tau_{j}^{\prime}\right)\right) .
\end{aligned}
$$

\section{Supervisory Design}

Before describing the supervisory design of the multi-robot system, let us give a brief overview of the process. Each robot starts with the Start state, then switches to the Searching block. In the Searching block, all robots will roam around the unknown environment with obstacle avoidance and search for the target object they must transport. Once the robots find the target object, the system will switch to the Formation block from the Searching block. The master robot will decide how many robots are needed to transport the target object cooperatively. After the robots form the appropriate formation, the system enters the Caging block. The cooperative robots will approach the target object and each stops at a suitable distance between it and a suitable marker to be identified on the target object. Finally, the robots will switch to Transportation block, where the target object will be transported to the desired location by the robots cooperatively.

After the overview of the system execution, we discuss the hybrid automata of the multi-agent robot system. The conceptual diagram of the underlying hybrid automaton is shown in Fig. 6(a) and Fig. 6(b).

It is noteworthy that some transitions will not change the discrete states, such as the transition in the "Wandering" state:

$$
\left\{\text { ChannelOK }\left(C_{m n}\right), \operatorname{Receive}\left(C_{m n}, \text { be_Slave }\right)\right\}
$$

which says that the variable be_Slave will be received by the robot ' $n$ ' when a message from the robot ' $m$ ' is available in the channel $C_{m n}$. Now, we will define the meaning of each state in the following:

\section{- Start :}

It indicates the beginning of the task at hand.

\section{- Wandering :}

At this state the WMM aims to move around within an unknown environment in a manner of random walk while avoiding collision with other WMMs and obstacles. So far several ways of performing the random path planning and complexity of these algorithms in a 2-D environment have been well studied. The wandering with random walk strategy will explore the unknown environment completely as time approaches infinity. Here, in order to speed up the response of the visual detection, we use "color" as the primary feature, i.e., every target object and every WMM is given a specific color. When such primary feature is detected by the vision algorithm, the WMM will switch to the "Searching" state. We will discuss the decision rules in next section. Otherwise, if one robot sends a request for cooperation from a master robot, the state of the former robot will switch to the "GoToArea" state.

- Searching :

When a WMM detects the primary feature of a target object or some other robot, it will then try to confirm whether a target object or some other WMM exist in the field of view. After the situation is confirmed, WMM tries to avoid collision with other robots no matter what the subsequent mission it will undertake. But if it is a target object which is confirmed, the robot state will switch to the "GoToLocation" state and then broadcast the request message to other robots for cooperation. If the target object no longer exists in the field of view, the robot state will readily switch back to the "Wandering" state. On the other hand, any robot in the 


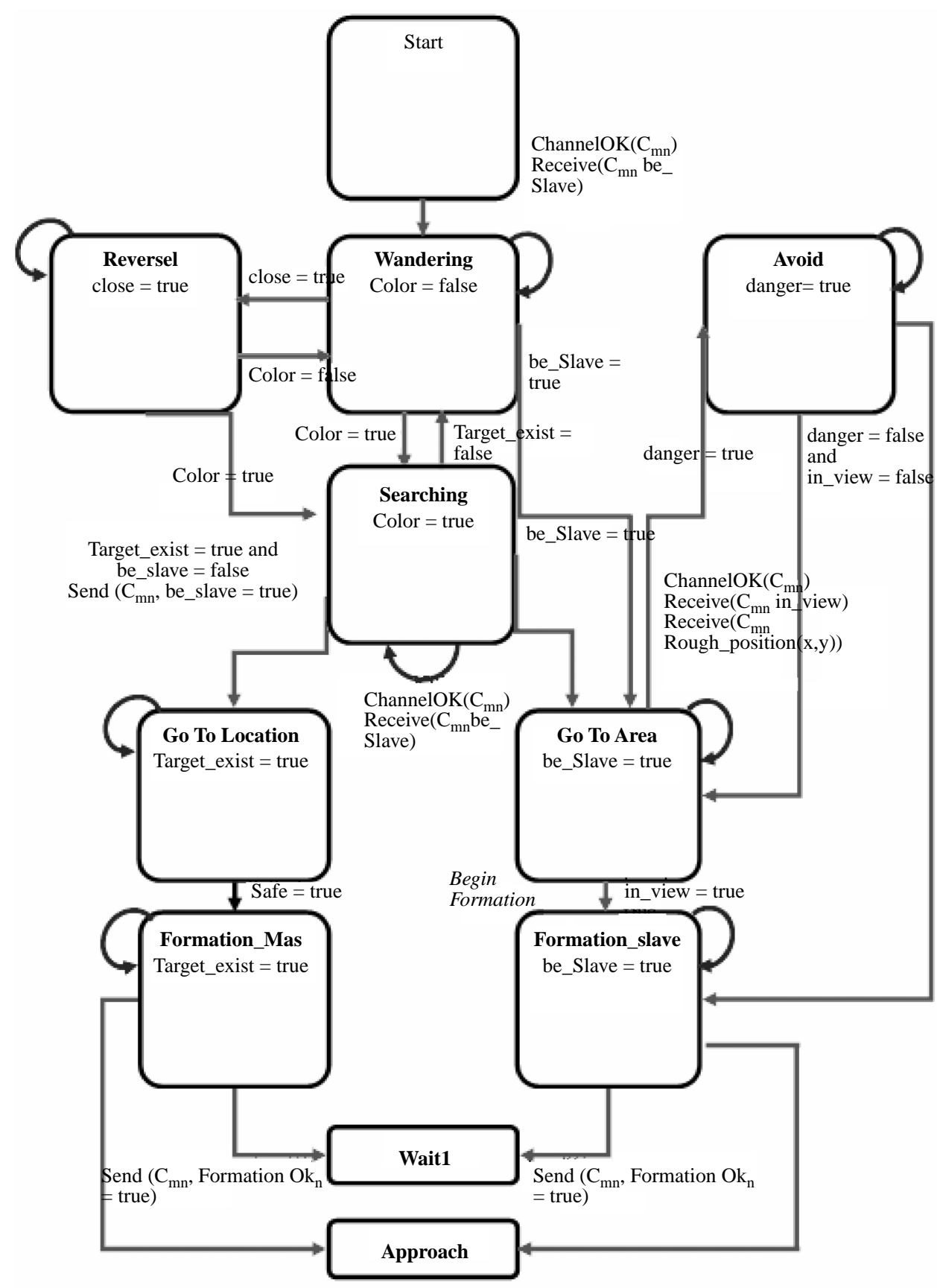

Fig. 6 (a) The conceptual diagram of underlying hybrid automaton

Searching state will listen to the message from WMM, too. If it receives the message for cooperation, its state will switch to the "GoToArea" state.

\section{- Reverse_1}

In order to avoid colliding with obstacles while robots wander in the unknown environment, the WMM will enter the "Reverse_1" state if obstacles are sensed. Also, the WMM is backed away slightly to avoid collision. The WMM will switch to the
Searching state if the image feature appears in the image plane; otherwise, it will turn back to the Wandering state.

\section{- GoToLocation :}

The WMM which finds the target object first will become the master and enter the "GoToLocation" state. Incidentally, it broadcasts the specific message to other one in request for cooperation while moving towards the target object. After it collects responses from some 


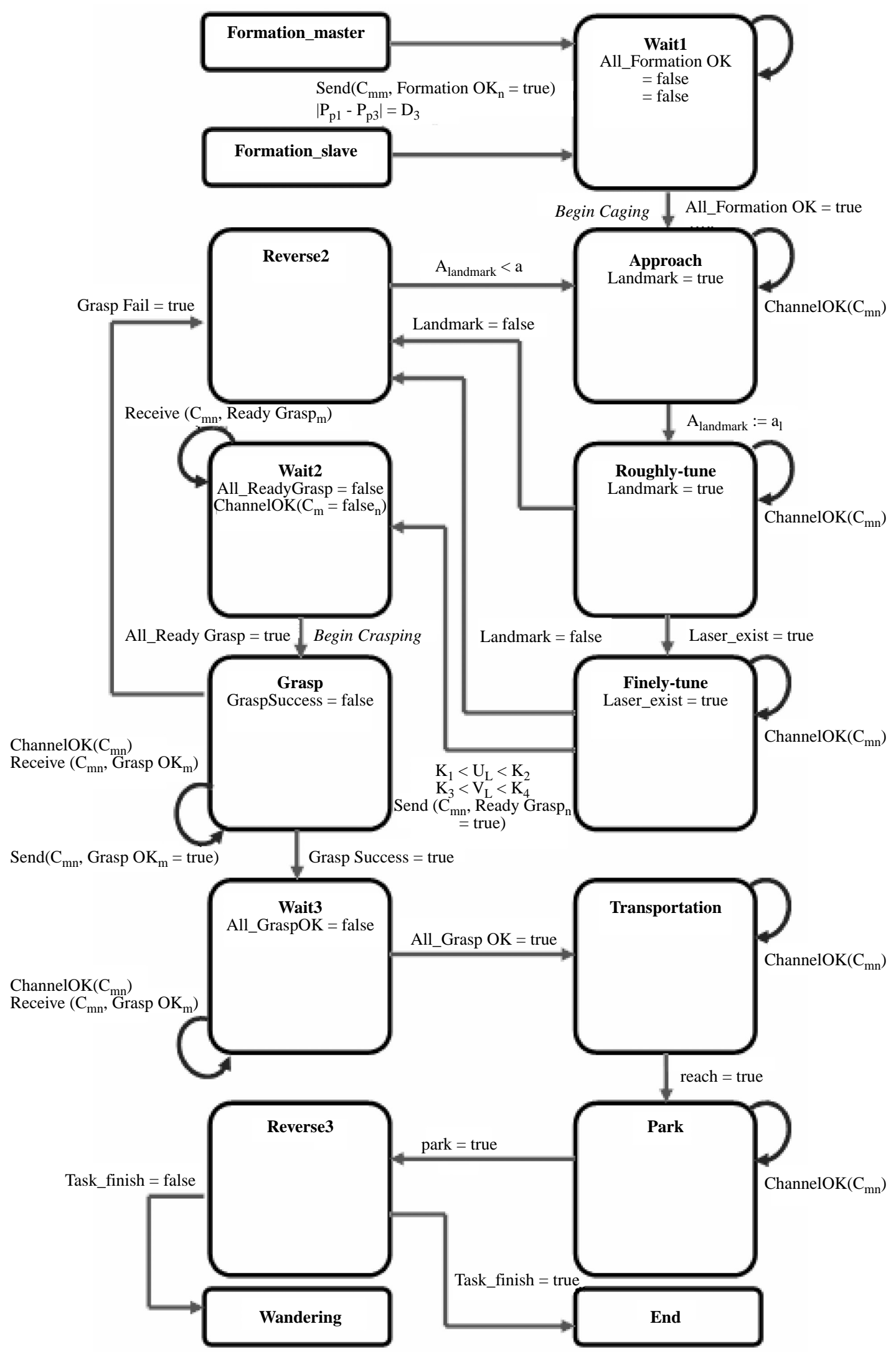

Fig. 6 (b) The conceptual diagram of underlying hybrid automaton 
other robots that can offer the help, it will then pick the most appropriate ones to form a cooperative team. When the master WMM can move towards the target object without any obstacle in front of it, the system mode will switch to the "Formation_Master" state. At the same time, the master WMM will also receive the message from other slave robots.

\section{- GoToArea :}

Every WMM that receives the massage from the master WMM to request for cooperation and is picked for help will be deemed as slave robots and enter the "GoToArea" state. Hence, it will assign one appropriate position for each of the slave robots, and these slave robots will try to move towards their assigned positions with ability to avoid obstacles. In other words, while slave WMMs are approaching their respective goals, they execute the function of obstacle avoidance when any obstacles are detected to be on their ways. Next, the aforementioned slave WMM will switch to the "Formation_slave" state when they are in the field of view of the master WMM. From this time on, the slave robots will obtain their positions relative to the master WMM. Meanwhile, every slave WMM receives the message from other slave WMMs to make sure that they will not collide with one another.

\section{- Formation_master :}

In this state, the master WMM broadcasts the request message to other robots. Slave WMMs are moving to their desired location individually while the master one moves towards to the target object. When the master WMM stops at a distance from the target object, it will send one message to the slave robots saying that it is ready to approach the target object. At the same time, it will receive the messages from these slave robots if they are ready to approach the target object and enter the "Wait_1" state.

\section{- Avoid :}

After a WMM receives the massage for cooperation request from the master WMM, it will move toward the goal position immediately. Note that WMMs may collide with obstacles during the process of approaching the goal position. Therefore, when the WMMs which are in the GoToArea state suffer the collision problem, the real action switches to the "Avoid" state. After the hazardous event is passed, GoToArea state will be restored from the Formation_Slave state.

\section{- Formation_slave :}

Any WMM which is in the GoToArea state will enter the "Formation_slave" state while it is being sensed by the master one. At that time, every slave WMM not only moves towards the target object but also to a specific position assigned from the master in order to form the required formation with other slave WMMs. After arriving at the goal location, it will send a message to the other slave WMMs to inform them that it is ready to approach the target object and then switch its state to "Wait_1" state from the current state.

\section{- Wait_1 :}

Each WMM in a certain cooperative team will receive messages from other robots and wait for other WMMs to arrive at their formation positions and then approach the target object simultaneously in this state. If the flags of all WMMs are set, then they will start approaching the target object and switch to the "Approach" state simultaneously.

\section{- Approach :}

In this state, the system will turn on the visual tracking mechanism so that the WMM will move towards the target object while trying to maintain visual contact with that target object. After the area of the target object in the CCD image matches some appropriate preset value $a_{1}$, then the state will transfer from "Approach" state to the "Roughly-tune" state.

\section{- Roughly-tune :}

If the WMM drives close enough to the target object, the state of "Roughly-tune" transfers to another state called "Finely-tune" state. Hereafter, the signal of the laser range finder will be turned on until this fine-tune state is ended. Here, the robot will enter "Reverse_2" state when it loses visual contact with the target object.

\section{- Finely-tune :}

When the image of the laser dot created by the laser beam shot at the target surface falls within some specific range around the center of the image which means that the equipped manipulator is currently at the right position for grasping, the mobile robot will stop and enter the "Wait_2" state followed by sending of a message to other WMMs. Likewise, the WMM will enter "Reverse_2" state when it loses visual contact with the target object.

\section{- Wait_2 :}

After the WMM stops in front of the target object at a suitable distance, it will wait to receive the 


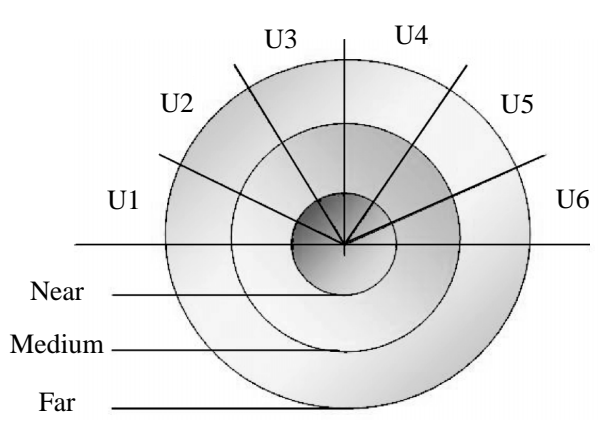

Fig. 7 The definition of ultrasonic working range

messages from the other robots. When all other cooperative WMMs are also in the "Wait_2" state, it will switch from "Wait_2" state to "Grasp" state and begin to grasp the target object cooperatively.

\section{- Grasp :}

This state indicates that all WMMs working in the cooperative state are ready to grasp the target object together. Besides, the manipulator on each WMM will move to the grasping configuration and execute the grasp motion. If the jump conditions are satisfied, WMMs will change to the "Wait_3" state while sending a message to other cooperative WMMs.

\section{- Wait_3 :}

After a WMM grasps the target object, it will wait for receiving the messages from other cooperating robots. When all of them are also in the "Wait_3" state, the system state will switch from "Wait_3" state to "Transportation" state and then begin to transport the target object cooperatively.

\section{- Transportation :}

In this state, the WMM will transport the target object cooperatively and switches to the Park state when they reach the destination.

\section{- Park :}

After reaching the destination, the cooperative robots will put the target object down and enter the "Reverse_2" state where all WMMs are backed sufficiently away from the target object.

\section{- Reverse_2 :}

After failure to grasp the object, which is detected by the CCD camera, or suffering from a loss of visual contact at either rough-tune or fine-tune

\begin{tabular}{|cc|c|c|c|c|c|}
\hline $\begin{array}{c}\text { Ultrasonic } \\
\text { Distance }\end{array}$ & U1 & U2 & U3 & U4 & U5 & U6 \\
\hline Near & LR & LR & B & B & SL & LL \\
\hline Midium & SR & SR & A & A & SL & SL \\
\hline Far & SR & SR & LA & LA & SL & SL \\
\hline
\end{tabular}

Fig. 8 Fuzzy rules table for obstacle avoidance

states, the Reverse_l state must be invoked immediately, i.e., the WMM will be backed slightly away to be ready to access the "Approach" state again.

\section{- Reverse_3 :}

After successfully grasping the target object, the remaining mission will be progressively completed. This state will be executed so that WMMs will be backed sufficiently away from the previous target object and the manipulator will get back to Home configuration so that the "Wandering" state will be accessed again.

\section{- End :}

When all the tasks are finished, the "End" state will be triggered.

From Fig. 7, the hunting behavior starts with assigning all variables with initial values and transferring to the wander mode. At that time, the obstacle avoidance flag sets to true that helps the mobile manipulator navigate in an unknown environment. Consequently, a fuzzy system is designed to avoid obstacles when the WMM searches through the environment. The target object and rule table is illustrated in Figs. 7 and 8.

In the rule table, LR denotes "Large Right" and SR is "Small Right" and B is "Reverse" and A is "Ahead" and LA is "Large Ahead" and SL is "Small Left" and LL is "Large Left".

Thus, the major processing flow of obstacle avoidance we formulate is as follows:

Step 1: Poll the signal from six ultra-sounds.

Step 2: Fuse signals with Fuzzy algorithm.

Step 3: Control the heading angle of the WMM.

The obstacle avoidance behavior is always true in the state of "W" until the state switches to another mode. In the state of "A", the behavior of obstacle avoidance will be activated by one of the six distances measured by ultra-sound sensors if the distance is short enough relative to our safty range. 


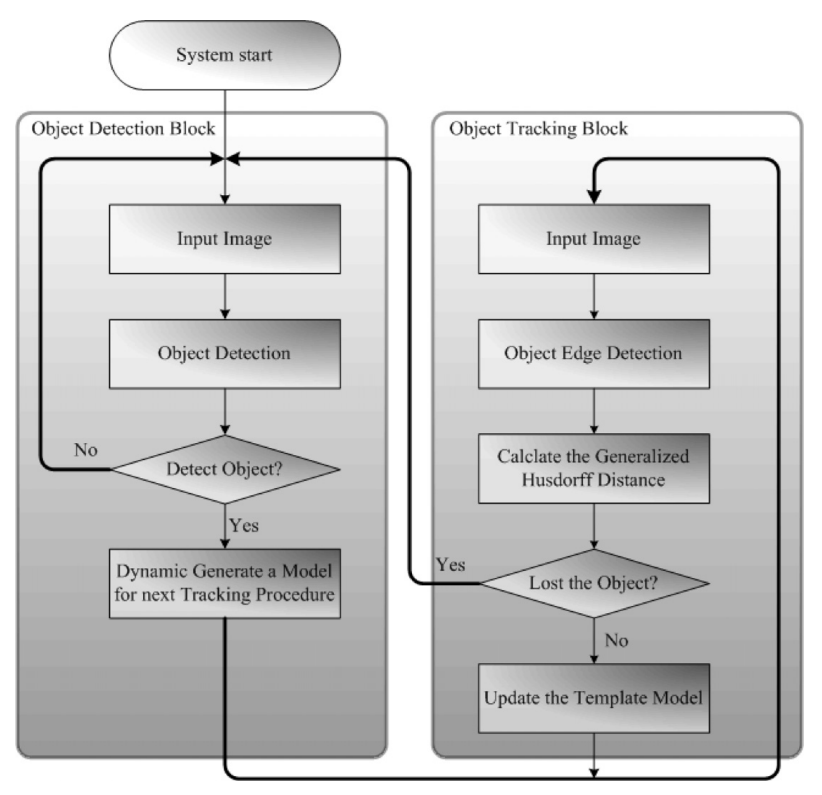

Fig. 9 Visual sensing system

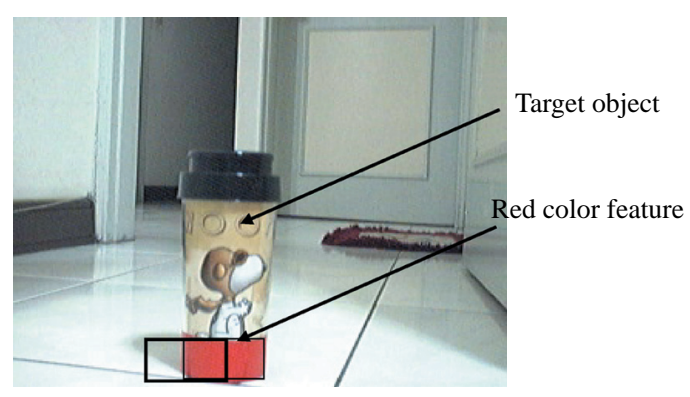

Fig. 10 Target object with red color feature

\section{SUB-SYSTEMS}

\section{Vision System}

In application, the mobile manipulator must confirm if a potential object image that appears in the field of view of the mobile vehicle is a target object or some other mobile manipulator, and the detection flow chart is shown in Fig. 9. We use "color" as the primary feature, i.e. every target object and every mobile robot is given a specific color for identification. One example of the target object with red color feature is shown in Fig. 10.

There are many factors such as vibration of the CCD camera, shadow resulting from the light source, and external noise disturbance will all influence the quality of input images. In order to reduce the noise interference, we execute the Gaussian filtering operator as shown in Fig. 11 to minimize the influence before further processing. The digital kernel is shown as follows:

After reducing the noise, we detect if the "color"

$\frac{1}{159}$\begin{tabular}{|c|c|c|c|c|}
\hline 2 & 4 & 5 & 4 & 2 \\
\hline 4 & 9 & 12 & 9 & 4 \\
\hline 5 & 12 & 15 & 12 & 5 \\
\hline 4 & 9 & 12 & 9 & 4 \\
\hline 2 & 4 & 5 & 4 & 2 \\
\hline
\end{tabular}

Fig. 11 Mask of the Gaussian filter $(\sigma=1.4)$

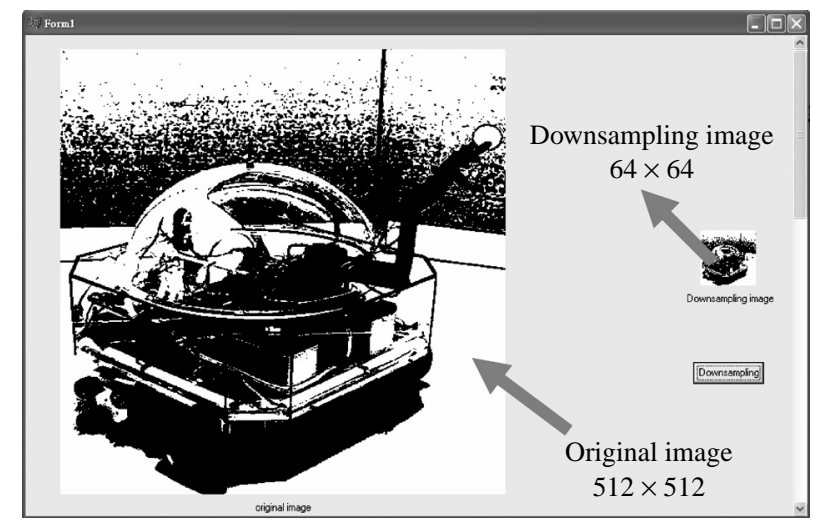

Fig. 12 Downsampling example

feature exists in the CCD camera image. If such "color" feature appears, we extract information $h$ and $w$ from this feature, where $h$ is height and $w$ is width of the feature. Subsequently, we will detect existence of the target associated with the color feature through template matching method.

We first downsample the template from the initial size to an appropriate size. For example: there is one template image whose size is $512 \times 512$ pixel $^{2}$ in Fig. 12. The downsampled template image using $8 \times 8$ blocks as a unit is also shown in Fig. 12. After that, we execute template matching process via Sum-ofAbsolute Differences (SAD) method.

If a target object or some other robot is confirmed in the CCD image, the mobile manipulator will keep tracking the target object or some other robot. But if it is some other situation which is confirmed, the mobile manipulator will keep detecting whether the color feature appears in the input image or not.

The flow chart of the object detection part is shown in Fig. 13 and the processing steps are depicted as follows:

(1) Remove noises from the input image by applying the Gaussian filter operator;

(2) Determine whether the "color" feature appears in the CCD camera image;

(3) Extract information $h$ and $w$ from the feature;

(4) Downsample the template from initial size to an 

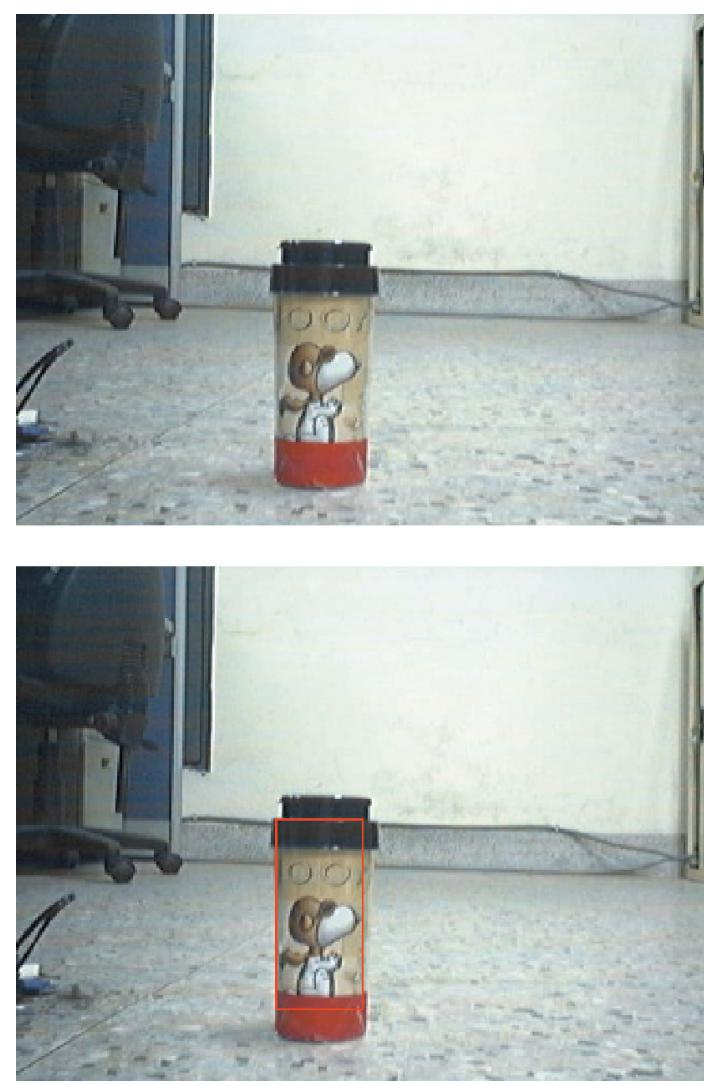

Fig. 13 Example of finding target object

appropriate size;

(5) Execute template matching process via SAD;

(6) Check if any target object or some other robot which appears in captured image or not.

\section{Laser Range Finder}

It is necessary to use the laser beamer and the vision system together to form an auxiliary device to help to precisely identify the distance parameters about some target object in front of the WMM so that the manipulator can be configured to successfully grasp the object. From the following figure (Fig. 14), one can see such setup, where $\alpha$ denotes a camera tilt angle, $H$ is the distance from CCD to ground, $d$ is the distance between the laser and the object, $V$ is a vertical location of the laser $\operatorname{dot} c$ in the camera image, $V_{0}$ is a initial value, then $d$ can be calculated by the formula :

$$
d=\left[H-K_{v}\left(V-V_{0}\right) \cdot \tan \alpha\right.
$$

where $K_{v}$ denotes a scalar constant.

\section{Wireless Communication}

For every WMM to execute control via (wireless)

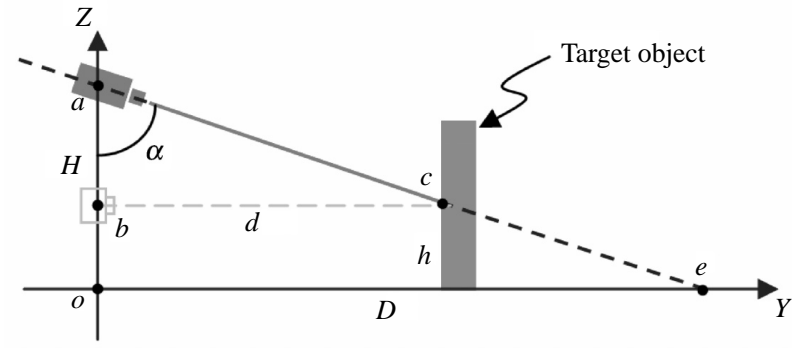

Fig. 14 A laser device as an auxiliary distance finder

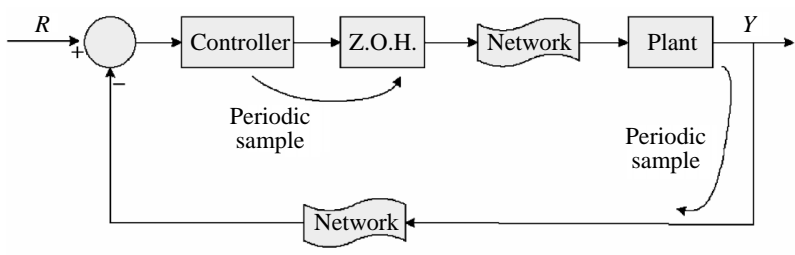

Fig. 15 Typical control system

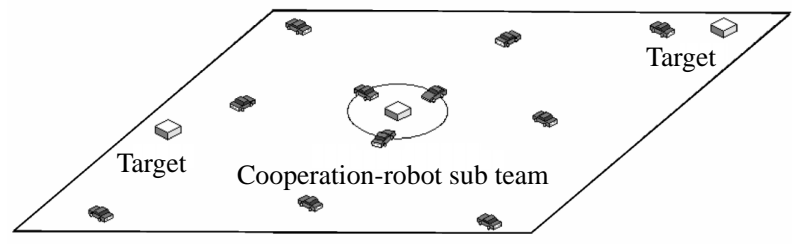

Fig. 16 Cooperative sub-team

communication network, the performance is always challenged by how real-time the needed series of data or information packets can be received. In other words, the performance will depend on the bandwidth whish is allocated to this particular WMM. In general, there will be no guarantee to any WMM to acquire the necessary bandwidth, but through some particularly identified communication channel with higher priority, say, WMM $i$ currently talks to $\mathrm{WMM} j$ intensively for trying to accomplish a task, a network controller can be designed to specifically allocate more bandwidth to this channel. Fig. 15 is a typical control system with network transmission.

In our system, every WMM is equipped with a wireless communication device which allows it to transmit any information or data packets to any other WMM. Generally speaking, when the robot calls for more network resource, the network controller allocates higher bandwidth to meet their demands. Hence, before accepting requests from each of robots, the central manager of the network evaluates the request whether the resource of allocation exceeds the limitation of the bandwidth, which can be half or a fraction less than one of the total network resource with our well designed configuration. If the demands, in addition, exceed the limit of resource allocation, the 


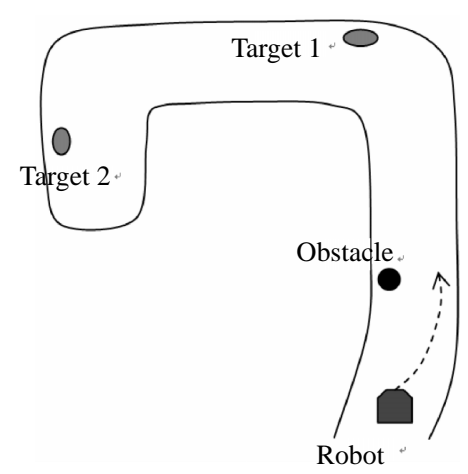

Fig. 17 Experiment scenario

controller rejects its request and sends back a message to the robot. This kind of admission control makes a robust balance between each of sub-teams of the multi-agent robot system. In Fig. 16, when a subteam of the multi-agent robot system groups together to co-work with limited network resource, it is necessary for every member of the team to conform to the total allocated bandwidth. At the same time, other sub-teams may search for other interesting targets given by their assigned tasks but sharing only the left network resource.

\section{EXPERIMENT AND SIMULATION RESULTS}

The experimental environment and devices are introduced first in the section. The intention here is to show that the multi-agent robot system whose control is built upon the hybrid automata approach can successfully demonstrate the non-blocking, safety, and goal reaching properties. However, more theoretical study and justifications of existence of these properties are currently under way.

\section{Scenario and Task}

As shown in Fig. 17, the size of the room for the experiments is $4 \times 5$ meter $^{2}$. A tunnel consists of two corners and two targets is arranged in the room. The WMM developed at NTU, called The Treasure Hunter (TTH), starts to wander from the starting point $(0,0,0)$ while turning on the collision avoidance mechanism. In this mode, the TTH will seek and lock in the target, in parallel with the functioning of collision avoidance mechanism. When the TTH finds the target via the CCD camera, it disables the collision avoidance mechanism and goes into tuning mode to approach to the target. During the tuning mode, the TTH tries to lock the target at the center of image. After approaching the goal closely enough, the TTH starts to grasp the target slowly. After all targets are
Table 2 Hardware architecture

\begin{tabular}{cc}
\hline Device & Descriptions \\
\hline Wireless LAN & 3Com $802.11 \mathrm{~g}$ USB Adapter \\
Ultrasonic Sensor & Polaroid $\times 6$ \\
Camera & Logitech QuickCam VGA \\
Manipulator & Synax \\
Batteries & Yuasa $12 \mathrm{~V} \mathrm{DC} \times 2$ \\
Main Computing & Advantech Pentium III \\
System & $-900 \mathrm{Mhz}$ \\
& PC/104 Module \\
\hline
\end{tabular}

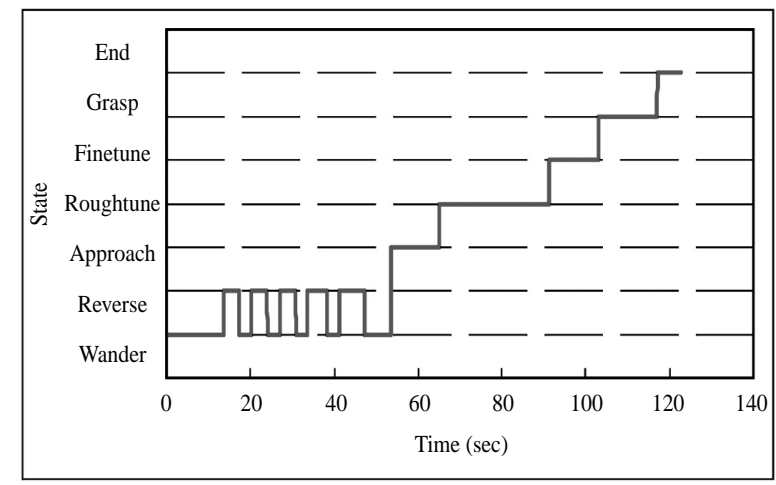

Fig. 18 An execution of hybrid automaton

grasped successfully, we will conclude that the designated mission is fully accomplished.

\section{Device Specification}

Following the hybrid automaton, we designed a mobile robot system with the hardware architecture as specified in Table 2 . We set each device on the mobile base made of aluminum sized $450 \mathrm{~mm} \times 450$ $\mathrm{mm}$; and the height of this robot is $500 \mathrm{~mm}$. Our robot totally weighs 11.2 Kilogram.

All of functions of WMM such as collision avoidance, target searching and tracking, and object grasping are contributed by the integration of the ultrasonic module, the vision system, the manipulator, and the computer system. Moreover, the two main processors are installed on board dedicated to real-time vision processing, on-line model construction of the dynamic environment, obstacle avoidance, cooperation with peer robots, and emergency tele-operation via Wireless LAN.

\section{Simulation}

Figure 18 shows the time history for state 


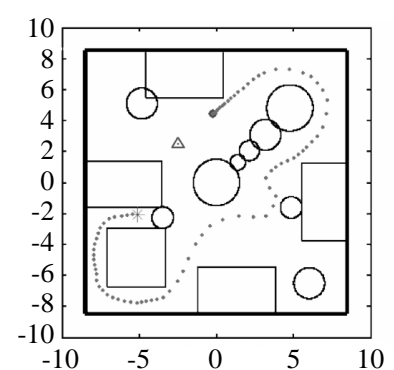

(a)

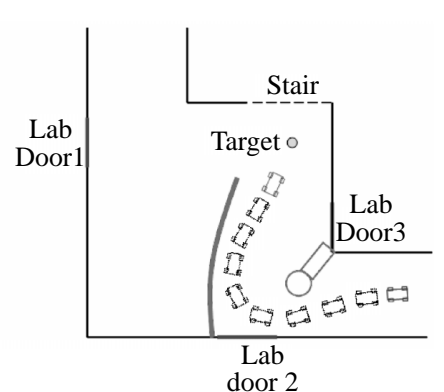

(b)

Fig. 19 Simulation and experimental results
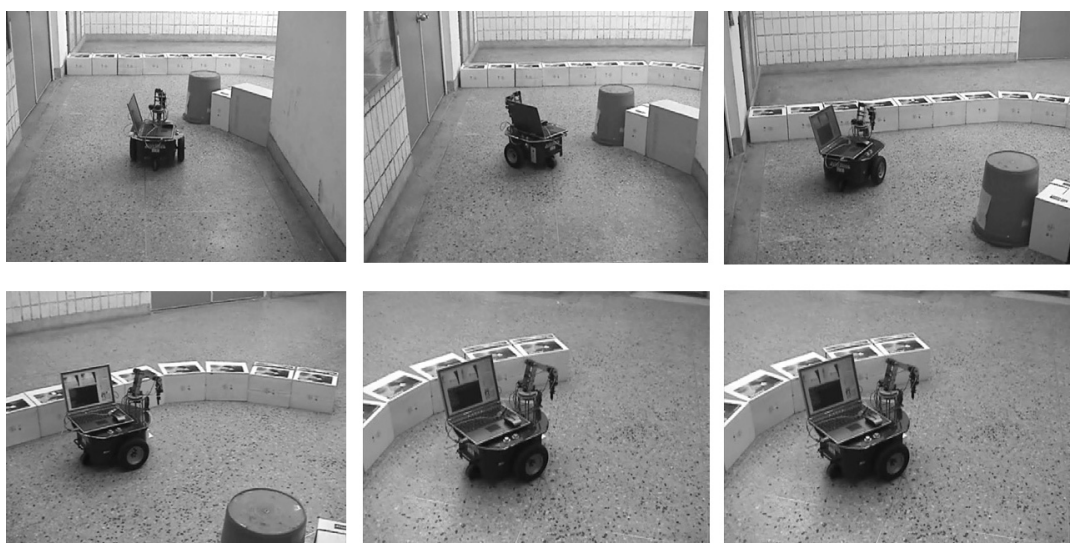

Fig. 20 Movements to avoid obstacles in series

switching in a treasure hunting procedure. Initially, the robot TTH wanders to explore the uncertain environment. Soon after that, the robot switches between two states, wandering state and reverse state, because it first goes to the corner, gets restricted, and tries to escape.

When the TTH discovers any target, the wandering state will switch to the approach state. Following the supervisory control mechanism, it will then switch into rough-tune and fine-tune states in series. $O$ ne can easily conclude that the grasping state will be reached in the figure, and the TTH will grasp the target successfully at the end.

\section{Searching for Target Object}

First of all, there is a mobile robot and a target object in the indoor environment. The robot is to look for object after extensive searching. The initial position of the mobile robot is marked as a star, whereas the target object is marked by a red triangle. After the experiment, the overall path trajectory is plotted in Fig. 19(a). It is true that the mobile robot wanders in the environment without collision and approaches to the target object successfully.
Tracking experiments are shown as Fig. 20. When the robot moves with time, sensing information from an ultrasonic system generates virtual force between every control period. At the end, the robot arrives at the location close enough to the desired target to facilitate subsequent manipulator grasping.

The robot successfully identifies the target object and will grasp the target object eventually which is displayed in Fig. 19(b).

\section{CONCLUSIONS}

In this paper, a novel hybrid system approach to design a control architecture for a WMM was proposed and extended into an HMAS. The resulting capability facilitates WMM to search for a target and grasp it within an unknown indoor environment. Initially, a novel hybrid system model was developed which captures the continuous dynamics of the WMM and the event-driven collision-avoiding goal seeking process. Next, a two-level control architecture is devised for the WMM system, where the first level mainly handles a continuous state control subject to sensor feedback signals, whereas the second level is 
responsible for providing a well performed supervisory control that can manipulate all available surrounding resources to successfully accomplish the aforementioned mission. In the Advanced Control Lab of Dept. of Electrical Engineering at National Taiwan University (NTU), an actual WMM was built, named The Treasure Hunter (TTH), which is able to hunt treasures in an unknown environment. To demonstrate the effectiveness of the proposed approach, both simulation through Webots and experiment in NTU have been done and their results were provided.

\section{ACKNOWLEDGMENTS}

This research is sponsored by National Science Council, R.O.C., under both Grant No. NSC-94-2213E-002-002 and Grant No. NSC94-2752-E-002-007PAE. The authors would like to send thanks to $\mathrm{Yu}$ Po Hsu, one of the fellow students of the Advanced Control Lab. at the same department, for his technical discussions as well as contributions.

\section{REFERENCES}

Chang, C. F., Huang, C. I., and Fu, L. C., 2004, "Nonlinear Control of Wheeled Mobile Robot with Nonholonomic Constrain," Proceedings of IEEE Conference Systems, Man, and Cybernetics, Vol. 6, pp. 5404-5410.

Altafini, C., 2004, "Redundant Robotic Chains on Riemannian Submersions," IEEE Transactions on Robotics and Automation, Vol. 20, No. 2, pp. 335340.

Ozvern, C. M., and Willsky, A. S., 1991, "Output Stabilizability of Discrete Event Dynamic Systems," IEEE Transactions on Automatic Control, Vol. 36, No. 8, pp. 925-935.

Nett, E., and Schemmer, S., 2003, "Reliable RealTime Communication in Cooperative Mobile Application," IEEE Transactions on Computers, Vol. 52, No. 2, pp. 166-180.

Durfee, E. H., Lesser, V. R., and Corkill, D. D., 1989, "Trends in Cooperative Distributed Problem Solving," IEEE Transactions on Knowledge and Data Engineering, Vol. 1, No. 1, pp. 63-75.

Mondada, F., Gambardella, L. M., Floreano, D., Nolfi, S., Denebourg, J. L., Dorigo, M., 2005, "The Cooperation of Swarm-Bots," IEEE Robotics and Automation Magazine, Vol. 12, No. 2, pp. 21-28.
Kolmanovsky, I., and McClamroch, N. H., 1995, "Developments in Nonholonomic Control Problems," IEEE Control Systems, Vol. 15, No. 6, pp. 20-36.

Lygeros, J., Johansson, K. H., Simic, S. N., Zhang, J. and Sastry, S. S., 2003, "Dynamical Properties of Hybrid Automata," IEEE Transactions on Automatic Control, Vol. 48, No. 1, pp. 2-17.

Passino, K. M., Michel, A. N., and Antsaklis, P. J., 1994, "Lyapunov Stability of a Class of Discrete Event Systems," IEEE Transactions on Automatic Control, Vol.39, No. 2, pp. 269-279.

Korayem, M. H., and Ghariblu, H., 2003, "Maximum Allowable Load on Wheeled Mobile Manipulators Imposing Redundancy Constraints," Robotics and Autonomous Systems, Vol. 44, pp. 151159.

Kumar, M., and Grag, D. P., 2004, "Sensor-Based Estinmation and Control of Forces and Moments in Multiple Cooperative Robots," Transactions of the ASME, Vol. 126, pp. 276-283.

Fan, M., Tan, Y., and Whinston, A. B., 2005, ”Evaluation and Design of Online Cooperative Feedback Mechanisms for Reputation Management," IEEE Transactions on Knowledge and Data Engineering, Vol. 17, No. 2, pp. 244-254.

Zhang, M., and Tarn, T. J., 2003, ”A Hybrid Control Strategy for Nonlinear and Uderactuated Mechanical Systems," IEEE Transactions on Automatic Control, Vol. 48, No. 10, pp. 1777-1782

Murray, R. M., and Sastry, S. S., 1993,"Nonholonomic Motion Planning: Steering Using Sinusoids," IEEE Transactions on Automacit Control, Vol. 38, No. 5, pp. 700-716.

Brockett, R. W., 2003, "Pattern Generation and the Control of Nonlinear Systems," IEEE Transactions on Automatic Control, Vol. 48, No. 10, pp. 1699-1711.

Burgard, W., Moors, M., Stachniss, C., and Schneider, F. E., 2005, "Coordinated Multi-Robot Exploration," IEEE Transactions on Robotics, Vol. 21, No. 3, pp. 376-386.

Kemeny, Z., 2003, "Redundancy Resolution in Robotics Using Parameterization though Null Space," IEEE Transactions on Industrial Electronics, Vol. 50, No. 4, pp. 777-783.

Manuscript Received: Nov. 05, 2005 and Accepted: Dec. 28, 2005 